\title{
La peste, les dieux et les hommes : cheminements d'une tradition
}

Danielle van Mal-Maeder

\section{(2) OpenEdition \\ 1 Journals}

Édition électronique

URL : http://journals.openedition.org/edl/276

DOI : $10.4000 /$ edl. 276

ISSN : 2296-5084

Éditeur

Université de Lausanne

\section{Édition imprimée}

Date de publication : 15 mai 2010

Pagination : $39-60$

ISBN : 978-2-940331-22-2

ISSN : 0014-2026

\section{Référence électronique}

Danielle van Mal-Maeder, «La peste, les dieux et les hommes : cheminements d'une tradition », Études de lettres [En ligne], 1-2 | 2010, mis en ligne le 15 mai 2013, consulté le 18 décembre 2020. URL : http:// journals.openedition.org/edl/276 ; DOI : https://doi.org/10.4000/edl.276 


\section{LA PESTE, LES DIEUX ET LES HOMMES CHEMINEMENTS D'UNE TRADITION}

L'étude de la tradition classique a pour objet de retracer les cheminements de la transmission et de la réception des textes grecs et latins. Elle enrichit notre perception de l'Antiquité en l'ouvrant sur d'autres paysages temporels. Cet article explore l'idée que la pérennité des œuvres antiques dépend des lectures toujours renouvelées qu'on en fait à différentes époques et que chaque lecture active dans le texte un sens qui correspond au contexte socio-culturel dans lequel elle est réalisée. Partant d'un poème chrétien du $\mathrm{IV}^{\mathrm{e}}$ siècle de notre ère, notre parcours nous conduira à remonter le temps jusqu'à Virgile, Lucrèce puis Thucydide, évoquera au passage le rôle de l'école dans le traitement littéraire de la peste, avant de nous ramener à l'époque moderne.

Every reading is different from every other reading; once again, there is no text-in-itself, but only a series (potentially endless?) of competing (or complementary) readings. Is a poem, in other words, better thought of as an event (in time) than as a thing?

Charles Martindale, Redeeming the text, p. 18.

L'histoire de la tradition classique débute dans l'Antiquité. Les Grecs et les Romains avaient leurs classiques et un canon soumis à certaines variations selon les goûts et les âges. Au dixième livre de l'Institution oratoire, un ouvrage consacré à l'éducation des jeunes Romains, Quintilien dresse une liste d'auteurs grecs et latins dont il recommande la lecture pour développer l'éloquence et qu'il conseille d'imiter en tentant de le surpasser ${ }^{1}$. C'est

I. Quintilien, Institution oratoire, X, 1, 20-X, 2, 28. Sur le classicisme antique, on consultera par exemple E. R. Curtius, La littérature européenne, p. 389-401; 
en grande partie selon ce principe de l'imitation-émulation, fondement de l'apprentissage scolaire et de la création littéraire, que se développe dans l'Antiquité une tradition classique. Ce principe, qui implique à la fois la recherche de la ressemblance et de la différence - l'imitateur doit s'efforcer d'égaler son modèle, voire de le surpasser - a pour effet d'instaurer entre les textes un dialogue que le lecteur antique, nourri de cette culture, savait entendre. Pour nous, Modernes, qui ne possédons plus que des pans du trésor littéraire antique, ce dialogue intertextuel est beaucoup plus difficile à saisir. Les relations que nous établissons entre les textes relèvent souvent de la supposition; elles dépendent d'interprétations tout à fait subjectives.

Le premier texte que j'évoquerai pour ma démonstration, dans un cheminement qui remontera le fil du temps, est un poème bucolique tardif, intitulé De la mort des boufs (De mortibus boum). Il est l'œuvre d'un certain Severus Sanctus Endelechius, rhéteur de son état, c'est-à-dire professeur de rhétorique, qu'on identifie avec un ami de Paulin de Nole et qui a peut-être dirigé une école de rhétorique à Rome, au $\mathrm{IV}^{\mathrm{e}}$ siècle de notre ère ${ }^{2}$. Ce poème, formé de trente-trois strophes en vers asclépiades, met en scène des pâtres en train de dialoguer. Le premier, Bucolus («le Bouvier »), relate au second, Aegon, la mort de son troupeau, décimé par une épidémie. Arrive un troisième berger, nommé Tityre. Son troupeau a été épargné. Interrogé par ses compagnons, il leur répond que c'est le signe de la croix qui a éloigné d'eux le terrible mal:

Hoc signum mediis frontibus additum

cunctarum pecudum certa salus fuit.

Sic uero deus hoc nomine praepotens

Saluator uocitatus est.

Fugit continuo saeua lues greges,

Morbis nil licuit.

M. Citroni, "Percezioni di classicità nella letteratura latina», ainsi que le volume Le classicisme à Rome aux I ${ }^{\text {ers }}$ siècles avant et après J.-C., passim; voir aussi, à propos de Virgile, les réflexions de Ch. Martindale, "Introduction: "The classic of all Europe" ".

2. Voir W. Schmid, "Tityrus Christianus. Probleme religiöser Hirtendichtung an der Wende vom vierten zum fünften Jahrhundert", p. 120-128; M. Barton, Spätantike Bukolik zwischen paganer Tradition und christlicher Verkündigung. Das Carmen De mortibus boum des Endelechius, p. 16-17; B. Effe et G. Binder, Antike Hirtendichtung. Eine Einführung, p. 141-146. 
Ce signe, inscrit au milieu de leur front, assura le salut de toutes mes bêtes. Ainsi, il porte bien son nom, le Dieu tout puissant que l'on appelle le Sauveur. Aussitôt cette peste cruelle a fui mon troupeau; aux maux, rien ne fut permis ${ }^{3}$.

La forme du poème - un chant à plusieurs voix -, le nom des bergers Aegon et surtout Tityre signalent le modèle: ce sont les Bucoliques de Virgile, qui ne cessent d'inspirer les poètes de l'Antiquité tardive ${ }^{4}$. Plusieurs échos renvoient en particulier à la première Bucolique, qui confronte le destin inégal de deux bergers. L’un deux, Mélibée, s'est vu confisquer son domaine au profit d'un soldat vétéran; l'autre, Tityre, a gardé le sien par la volonté d'un dieu, dit-il; un dieu que la suite du poème invite à identifier comme étant Octave, le futur empereur Auguste:

O Meliboee, deus nobis haec otia fecit:

namque erit ille mihi semper deus; illius aram

saepe tener nostris ab ouilibus imbuet agnus.

Ille meas errare boues, ut cernis, et ipsum

ludere quae uellem calamo permisit agresti.

O Mélibée, c'est à un dieu que nous devons ces loisirs; car Il sera pour moi toujours un dieu; son autel, une tendre victime, un agneau de nos bergeries, souvent l'ensanglantera. Grâce à Lui, mes génisses ont le droit de paître en liberté, comme tu vois, et moi-même celui de jouer mes airs préférés sur un roseau rustique ${ }^{5}$.

3. Endelechius, De la mort des bøufs, 109-114. Le texte latin est celui de l'Anthologia latina I. 2, p. 334-339; la traduction est mienne.

4. Voir B. Effe et G. Binder, Antike Hirtendichtung. Eine Einführung, p. 126-159; R. Mayer, "Latin Pastoral after Vergil»; R. Green, "Refinement and Reappraisal in Vergilian Pastoral».

5. Virgile, Bucoliques, I, 6-10; voir le commentaire ad loc. de W. Clausen, $A$ Commentary on Virgil Eclogues, p. 31-32; A. Powell, Virgil the Partisan. A study in the re-integration of Classics, p. 192-193. Sur l'interprétation politique du recueil, voir Ch. Martindale, "Green politics: the Eclogues», avec références supplémentaires; R. J. Tarrant, "Poetry and power: Virgil's poetry in contemporary context»; A. Powell, Virgil the Partisan. A study in the re-integration of Classics, p. 181-225. Pour une analyse détaillée des rapports entre le poème d'Endelechius et la première Bucolique, voir M. Barton, Spätantike Bukolik zwischen paganer Tradition und christlicher Verkündigung. Das Carmen De mortibus boum des Endelechius, p. 73-97. 
On voit bien le renversement. Dans le poème d'Endelechius, le Dieu des chrétiens a remplacé Octave divinisé. Le Tityre chrétien peut désormais se targuer d'avoir été sauvé par un Dieu qui n'exige aucun sacrifice animal:

\footnotetext{
Non ullis madida est ara cruoribus nec morbus pecudum caede repellitur, sed simplex animi purificatio optatis fruitur bonis.
}

Son autel n'est pas inondé de flots de sang et l'on ne repousse pas la maladie en massacrant des bêtes, non: une âme purifiée et candide jouit des biens qu'elle désire ${ }^{6}$.

Cette réappropriation de Virgile, un auteur païen, ne doit pas surprendre. Le rôle important qu'ont joué les œuvres païennes dans l'émergence de la littérature chrétienne est un phénomène bien connu. En ce qui concerne Virgile, ce poète était très vite devenu l'un des classiques sur lesquels l'enseignement scolaire se fonda pendant de longs siècles. Dans la littérature chrétienne, il occupa une place particulière, en raison notamment de la quatrième Bucolique qui célèbre la venue d'un nouvel âge d'or lié à la naissance d'un enfant mystérieux: les chrétiens, depuis l'empereur Constantin, y virent l'annonce prophétique de la venue du Sauveur ${ }^{7}$. Indépendamment de cette interprétation allégorique qui fait de Virgile un prophète, Augustin ou Prudence ne se privent pas d'emprunter au poète ses vers pour étoffer la métaphore du Bon Berger ou de la brebis égarée ${ }^{8}$.

Endelechius se sert donc dans son poème de l'autorité conférée par l'œuvre de Virgile - et par sa voix. En effet, s'il met en scène à son tour le berger Tityre pour énoncer un message protreptique, c'est sans doute parce que Tityre avait été identifié par les commentateurs anciens et par les biographes de Virgile avec l'auteur des Bucoliques. Une confusion

6. Endelechius, De la mort des boufs, 117-120.

7. Voir A. Perutelli, «Bucolics», p. 60-61; R. J. Tarrant, «Aspects of Virgil's reception in antiquity», p. 70-71; Lactance, qui deviendra précepteur du fils de Constantin, cite déjà des vers de la quatrième églogue dans la peinture du paradis qu'il fait dans ses Institutions divines 7, 24, 11 .

8. Voir W. Schmid, "Tityrus Christianus. Probleme religiöser Hirtendichtung an der Wende vom vierten zum fünften Jahrhundert», p. 110-114, avec références. 
s'était produite entre la personne réelle du poète et la figure du berger, confusion que l'on retrouve encore fréquemment dans la littérature critique moderne ${ }^{9}$. Dans le poème d'Endelechius, l'invitation faite aux bergers de se convertir acquiert davantage de poids du fait qu'elle émane de Tityre, double de Virgile.

Mais un autre texte du poète de Mantoue sous-tend les vers d'Endelechius. Il s'agit des Géorgiques, en particulier du troisième chant qui se termine avec la description d'une épizootie, une épidémie qui avait décimé les troupeaux du Norique, dans les régions orientales des Alpes. Une comparaison entre les deux textes révèle plusieurs correspondances verbales qui signalent le dialogue et soulignent les différences ${ }^{10}$. La description de l'épizootie au livre III des Géorgiques s'insère dans un développement sur les maladies des animaux: "Je vais aussi t'apprendre les causes et les symptômes des maladies", nous dit le poète au vers 440 , employant une formule qui renvoie à un autre poème didactique, le $D e$ la Nature (De rerum natura) de Lucrèce, sur lequel je reviendrai plus loin ${ }^{11}$. Ces maladies sont innombrables, elles se glissent au milieu des troupeaux sans défense, surviennent parfois de façon imprévisible, frappent de façon fulgurante, comme l'illustre la description de l'épizootie norique. Les circonstances demeurent imprécises. On est dans un passé lointain et indéfini (quondam: III, 478) et les causes de l'épidémie ne sont pas précisées. Virgile s'attarde davantage sur les symptômes de la maladie qui touche tous les animaux domestiques et sauvages, terrestres et marins. A vrai dire, plus que les symptômes, le poète décrit les signes annonciateurs de l'arrivée d'un mal mystérieux contre lequel ni la médecine ni la religion ne peuvent rien. Ce sont d'abord les victimes sacrificielles qui meurent soudainement, au moment où elles vont être

9. Voir N. Holzberg, Vergil. Der Dichter und sein Werk; A. Powell, Virgil the Partisan. A study in the re-integration of Classics, p. 186; A. Perutelli, "Bucolics", p. 58-61, qui cite le commentaire de Servius à la première Bucolique: Et hoc loco Tityri sub persona Vergilium debemus accipere; non tamen ubique, sed tantum ubi exigit ratio; cf. déjà Martial, 8, 55, 8 qui identifie Tityre à Virgile. L'œuvre du poète a parfois donné lieu à des reconstructions biographiques regrettables, comme le portrait fasciste qu'en donne R. Brasillach (condamné à mort en février 1945) dans Présence de Virgile (1931): voir F. Cox, «Envoi : the death of Virgil», p. 327.

Io. Pour une analyse détaillée, voir M. Barton, Spätantike Bukolik zwischen paganer Tradition und christlicher Verkündigung. Das Carmen De mortibus boum des Endelechius, p. 97-131.

II. Virgile, Géorgiques, III, 440 morborum quoque te causas et signa docebo. 
immolées (III, 486-488); ou, quand le sacrifice a eu lieu, les entrailles se révèlent corrompues (III, 489-493). Ici, la description est générale, elle énumère les morts soudaines parmi les animaux, race par race (III, 494-497); là, Virgile s'attarde plus longuement sur le sort d'un animal, comme lorsqu'il décrit la mort subite d'un bœuf attelé à une charrue:

Ecce autem duro fumans sub uomere taurus concidit et mixtum spumis uomit ore cruorem extremosque ciet gemitus. It tristis arator maerentem abiungens fraterna morte iuuencum atque opere in medio defixa relinquit aratra.

Mais voici que, fumant sous la pesante charrue, le taureau s'effondre et vomit un sang mêlé d'écume, et pousse les gémissements de l'agonie. Triste, le laboureur s'en va dételer le jeune taureau affligé de la mort de son frère, et laisse sa charrue enfoncée au milieu du sillon ${ }^{12}$.

Cet épisode amène le poète à exprimer son indignation et à s'interroger sur le sens d'une existence consacrée au labeur:

Quid labor aut benefacta iuuant? Quid uomere terras inuertisse grauis?

Que leur sert d'avoir peiné et rendu des services? Que leur sert d'avoir retourné avec le soc la terre pesante? ${ }^{13}$

Les bœufs, comme les paysans, mènent une vie saine et mesurée, une vie sans excès, qui ne justifie pas un tel sort. Ces interrogations suggèrent l'idée de l'épidémie comme châtiment divin, un motif traditionnel remontant aux poèmes homériques et que l'on rencontre aussi dans l'Ancien Testament ${ }^{14}$. La description de l'épidémie culmine d'ailleurs avec l'évocation épique de la Furie Tisiphone, semant devant elle crainte et désolation ${ }^{15}$.

Le poème De la mort des boufs d'Endelechius s'inspire en partie des Géorgiques qu'il adapte, condense et développe selon son propos et en

\footnotetext{
I2. Ibid., III, 515-519.

I3. Ibid., III, 525-526.

I4. J. Grimm, Die literarische Darstellung der Pest in der Antike und in der Romania, p. 14-31.

I5. Virgile, Géorgiques, III, 551-558.
} 
fonction du genre de la poésie bucolique. Ces modifications concernent tant le fond que la forme. Le caractère général de la description virgilienne et le ton didactique du discours font place à un récit personnel. Nous ne sommes plus dans un passé lointain et indéfini. C'est la mort de ses propres bêtes que le berger Bucolus raconte à Aegon. On y retrouve l'épisode des deux bœufs attelés à une même charrue, mais l'incident est développé et le caractère anthropomorphe des animaux accentué:

Plaustris subdideram fortia corpora lectorum studio, quo potui, boum, queis mentes geminae, consona tinnulo concentu crepitacula, aetas consimilis saetaque concolor, mansuetudo eadem, robur idem fuit et fatum: medio nam ruit aggere par uictum parili nece. Mollito penitus farra dabam solo: largis putris erat glabea liquoribus, sulcos perfacilis stiua tetenderat, nusquam uomer inhaeserat: laeuus bos subito labitur impetu, aestas quem domitum uiderat altera. Tristem continuo disiugo coniugem, nil iam plus metuens mali.

Dicto sed citius consequitur necem, semper qui fuerat sanus et integer; tunc longis quatiens ilia pulsibus uictum deposuit caput.

J'avais attaché à la charrue mes bœufs, des bêtes puissantes, choisies avec tout le soin possible; ils avaient des pensées jumelles, leur clochette émettait en accord un tintement argentin; tous deux du même âge, tous deux de même couleur, ils avaient la même douceur, la même force; et ainsi de leur destin: car ils s'effondrèrent au beau milieu d'un tertre, paire terrassée par une mort pareille. Je semais le blé profondément dans le sol ramolli, les pluies abondantes avaient rendu la terre grasse; la charrue avait très facilement traversé les sillons, jamais le soc n'était resté croché. Le bœuf de gauche, brusquement, s'écroule, c'était la deuxième année qu'il avait été mis à l'attelage; son compagnon, accablé de tristesse, je le détache sans craindre 
d'autres malheurs. Mais plus vite qu'on ne saurait le dire, la mort s'empare de lui qui fut toujours sain et bien portant. Alors, les flancs secoués de longs spasmes, il laissa retomber sa tête, sans plus résister ${ }^{16}$.

Bucolus rapporte ensuite l'agonie du reste de son troupeau qu'il dépeint comme une famille: la vache, grosse d'un veau condamné à mourir en elle, les petits veaux gambadant dans l'herbe, soudainement terrassés ou tétant le lait empoisonné de leur mère malade, le taureau, enfin, père et époux des victimes. Le pathétique de ce récit vient de ce qu'il est fait non pas par une voix poétique externe, mais par le pâtre lui-même répondant aux questions de son collègue. Personnage anonyme chez Virgile, caractérisé par le seul adjectif tristis (III, 517), il se dote chez Endelechius d'un nom, d'une personnalité, et d'une voix propre. Cette transposition du genre de la poésie didactique à la poésie bucolique résulte en une dramatisation (au sens théâtral), comme si Endelechius, en "zoomant» sur ces vers, avait choisi d'en développer la valeur scénique.

Ainsi, la voix énonciative unique des Géorgiques cède la place au chant à deux et à trois voix de la bucolique. Cette mise en scène dialogique est comme le reflet du dialogue qui s'engage avec Virgile autour du rôle de la religion dans l'épizootie. Dans les Géorgiques, sacrifices et dévotion se révèlent inutiles face au mal. La raison en est donnée par le Tityre chrétien d'Endelechius: seul le Dieu des chrétiens, qui n’admet pas les sacrifices, peut répondre aux prières des bergers. Le renversement opéré a également pour effet d'effacer l'idée que la cause du mal puisse avoir une origine divine. Sur ce point, Endelechius s'accorde avec un autre poète profane qu'il connaissait sans doute, un troisième homme avec lequel Virgile avait lui aussi engagé un dialogue intertextuel.

C'est un fait bien établi que le poème De la Nature de Lucrèce est un modèle important pour les Géorgiques. Macrobe, déjà, au $\mathrm{V}^{\mathrm{e}}$ siècle de notre ère, dressait une liste de passages empruntés par Virgile à son prédécesseur ${ }^{17}$. La description de l'épizootie, en particulier, s'inspire largement de la fin du poème De la Nature, qui s'achève avec la

16. Endelechius, De la mort des boufs, 33-52.

17. Macrobe, Saturnales, VI, 2, 7: Ipsius uero pestilentiae, quae est in tertio Georgicorum, color totus et liniamenta paene omnia tracta sunt de descriptione pestilentiae quae est in sexto Lucretii. Cette observation est suivie d'une série de citations illustrant le rapport de filiation. 
description d'une épidémie ayant ravagé la ville d'Athènes autrefois (quondam: VI, 1138). Avant de s'insinuer dans la ville, le mal avait ravagé les campagnes, frappant les bergers et leurs bêtes. Je ne me risquerai pas à comparer les deux textes en détail : l'exercice a été effectué à maintes reprises, aboutissant à des interprétations parfois très divergentes ${ }^{18}$. Mais je relèverai tout de même certains points concernant le rôle des dieux, puisqu'il s'agit là d'un élément central sur lequel s'est construite une tradition autour du thème de la peste.

Le poème De la Nature, on le sait, se fonde sur la philosophie d'Epicure qui préconisait de bannir de l'âme humaine les peurs qui l'assaillent: peur de la mort, des dieux, des phénomènes naturels. Dans cette perspective, Epicure avait repris de Démocrite pour la développer la théorie physique de l'atomisme. Lucrèce expose à son tour les principes de cette philosophie, capable de libérer les hommes de la peur des dieux et de celle de la mort. Les livres V et VI traitent du monde, démontrant qu'il n'a pas de nature divine, qu'il est destiné à périr et que les dieux lui sont étrangers. Les phénomènes célestes et naturels, comme le cours du soleil et de la lune, les éclipses, le tonnerre, les éclairs, la foudre, les tremblements de terre, les maladies aussi et les épidémies, s'expliquent de façon rationnelle. Ils ne sont pas le fait d'interventions divines. Ainsi les maladies et les épidémies proviennent-elles de germes qui circulent en grand nombre dans l'air, se rencontrent parfois, corrompent le ciel et rendent l'atmosphère morbide. Selon Lucrèce, les dieux existent, mais ils ne s'occupent pas des hommes, ils vivent dans un monde distinct du nôtre. Les craintes superstitieuses n'ont d'autre fondement que l'ignorance des hommes. Les prières et les sacrifices sont inutiles:

Nec pietas ullast uelatum saepe uideri uertier ad lapidem, atque omnis accedere ad aras, nec procumbere humi prostratum et pandere palmas ante deum delubra, nec aras sanguine multo spargere quadrupedum, nec uotis nectere uota, sed mage pacata posse omnia mente tueri.

I8. Parmi d'autres, D. A. West, "Two Plagues: Virgil, Georgics 3, 478-566 and Lucretius 6, 1090-1286»; K. Freudenburg, "Lucretius, Vergil, and the Causa Morbi»; M. Gale, "Man and Beast in Lucretius and the Georgics", p. 421-424; eadem, Virgil on the Nature of Things: the Georgics, Lucretius and the Didactic Tradition, p. 45-48; A. Powell, Virgil the Partisan. A study in the re-integration of Classics, p. 230-231. 
La piété, ce n'est point se montrer à tout instant couvert d'un voile et tourné vers une pierre, et s'approcher de tous les autels; ce n'est point se pencher jusqu'à terre en se prosternant, et tenir la paume de ses mains ouvertes en face des sanctuaires divins; ce n'est point inonder les autels du sang des animaux, ou lier sans cesse des vœux à d'autres vœux; mais c'est plutôt pouvoir tout regarder d'un esprit que rien ne trouble ${ }^{19}$.

Ce sont ces principes qu'illustre le récit de l'épidémie d'Athènes, un récit terrifiant qui s'attarde sur la dégénérescence physique et psychologique des malades, lorsqu'ils sont assaillis par la crainte de la mort. Ce récit culmine avec la description des temples des dieux, encombrés des cadavres qu'on y avait entassés: image saisissante, qui dit l'inanité de la religion et prouve que les dieux sont bien loin. Le seul espoir de l'homme réside dans sa connaissance du monde et dans la sagesse transmise par celui dont Lucrèce parle comme d'un dieu: Epicure, l'Athénien, qui "par sa science, arrachant notre existence à des tempêtes si grandes, à des ténèbres si profondes, a su l'asseoir dans un calme si tranquille, dans une si claire lumière» $(\mathrm{V}, 10-12)^{20}$. Le drame des Athéniens, victimes de l'épidémie décrite à la fin du poème, est de ne pas avoir bénéficié de l'enseignement d'Epicure. C'est la conclusion qui s'impose, me semble-t-il, si on se souvient que le récit de Lucrèce est une récriture de la description par Thucydide de la "peste» d'Athènes, survenue en 430 avant notre ère, soit presque un siècle avant la naissance du philosophe.

Mais avant de poursuivre mon cheminement, j'aimerais souligner davantage ce qui apparaît comme une différence importante entre le poème de Lucrèce et celui de Virgile. Si l'auteur des Géorgiques s'inspire de son modèle, s'il l'imite, il s'y oppose en ce qui concerne le rôle joué par les dieux dans l'épidémie. Certains passages, nous l'avons vu, suggèrent l'idée d'une colère divine. La dimension épique de la description fait intervenir Tisiphone, la Peur et les Maladies comme des personnifications, des entités divines nées des craintes religieuses rejetées par le rationalisme de Lucrèce ${ }^{21}$. La présence des dieux s'exprime aussi dans des interventions plus personnelles qui rompent avec le ton presque objectif

19. Lucrèce, De la Nature, V, 1198-1203.

20. Ibid., V, 10-12: ... quique per artem / fluctibus e tantis uitam tantisque tenebris / in tam tranquillo et tam clare luce locauit.

2I. Virgile, Géorgiques, III, 551-558. 
de Lucrèce. Ainsi, en décrivant l'agonie des chevaux, brûlant de toutes les fureurs, Virgile s'exclame: "Dieux, traitez mieux vos fidèles et réservez cet égarement à leurs ennemis!» ${ }^{22}$ Les dieux sont d'ailleurs omniprésents dans les Géorgiques dont ils peuplent les campagnes, les pâturages et les forêts. Certes, ils guident les hommes dans leur labeur, mais ils les mettent aussi à l'épreuve en faisant surgir des obstacles comme les maladies. Cette différence par rapport à Lucrèce s'exprime dans un passage célèbre, emblématique du dialogue engagé avec son modèle:

Felix qui potuit rerum cognoscere causas, atque metus omnis et inexorabile fatum subiecit pedibus strepitumque Acheruntis auari! Fortunatus et ille deos qui nouit agrestis, Panaque Siluanumque senem Nymphasque sorores!

Heureux qui a pu connaître les principes des choses, qui a foulé aux pieds toutes les craintes, l'inexorable destin et tout le bruit fait autour de l'insatiable Achéron! Bienheureux aussi celui qui connaît les dieux champêtres, et Pan et le vieux Sylvain et les Nymphes sœurs! ${ }^{23}$

Et le poète de poursuivre en faisant l'éloge de la vie champêtre, respectueuse de la morale et des traditions. Si l'on admet, avec une partie de la critique, que les Géorgiques reflètent des éléments de la propagande idéologique d'Octave concernant le rétablissement des anciennes valeurs éthiques et religieuses, on comprend mieux la nécessité de transformer le discours lucrétien sur les dieux ${ }^{24}$. Dans son panthéon, Virgile accorde d'ailleurs une place d'honneur à Octave, seul capable de mettre fin aux guerres civiles et dont il annonce l'apothéose dès l'ouverture du premier chant des Géorgiques ${ }^{25}$.

La description de l'épidémie d'Athènes chez Lucrèce, je le disais, est une récriture de celle qu'on trouve chez l'historien grec Thucydide, au

22. Ibid., III, 513: Di meliora piis erroremque hostibus illum!

23. Ibid., II, 490-494. Ces vers sont commentés entre autres par M. Gale, Virgil on the Nature of Things: the Georgics, Lucretius and the Didactic Tradition, p. 9 et par $\mathrm{Ph}$. Hardie, "Lucretius and later Latin literature in antiquity», p. 115.

24. Voir Ph. Hardie, "Lucretius and later Latin literature in antiquity», p. 115-116; A. Powell, Virgil the Partisan. A study in the re-integration of Classics, p. 227-281. Sur le rôle des dieux dans les Géorgiques, voir M. Gale, Virgil on the Nature of Things: the Georgics, Lucretius and the Didactic Tradition, p. 58-78.

25. Virgile, Géorgiques, I, 24-42. 
deuxième livre de La guerre du Péloponnèse. Nous remontons encore un peu plus haut dans le temps, au $\mathrm{V}^{\mathrm{e}}$ siècle avant notre ère, à l'époque du conflit entre Sparte et Athènes. Comme pour Lucrèce et Virgile, les études comparant le texte de Thucydide et celui de Lucrèce ne manquent pas ${ }^{26}$. Le récit de la "peste» d'Athènes chez Thucydide s'insère dans son histoire de La guerre du Péloponnèse qu'il avait entrepris d'écrire dès le début du conflit. Nous sommes en 430, c'est l'été, les Lacédémoniens et leurs alliés occupent l'Attique lorsqu'une épidémie s'abat brusquement sur la ville. Il est traditionnel de parler de la "peste d'Athènes". En réalité, ce ne fut probablement pas la maladie infectieuse transmise par les rats, la peste noire ou peste bubonique que le terme évoque dans notre imaginaire. Le mot grec $\lambda$ oupós s'emploie pour toute infection épidémique, de même que pestis en latin; comme en français, ces termes revêtent parfois le sens figuré de "fléau", "calamité». Les spécialistes de l'histoire de la médecine ont émis diverses suppositions sur la nature de l'épidémie: rougeole, variole, typhus, fièvre jaune, virus Ebola? Le doute subsiste $^{27}$. Quoi qu'il en fût, le mal était inconnu: "On n’avait nulle part souvenir de rien de tel comme fléau ni comme destruction de vies humaines ${ }^{28}$. Face à ce mal nouveau, inconnu, les médecins ne pouvaient rien. De même, les supplications dans les sanctuaires ou le recours aux oracles, tout restait inefficace. Pour finir, ajoute Thucydide, «ils y renoncèrent, s'abandonnant au mal ${ }^{29}$. La suite du récit s'attarde sur la description des symptômes de la maladie, de son caractère contagieux, ainsi que sur la dégradation morale que le fléau entraîna dans la cité:

26. E.g. J. Grimm, Die literarische Darstellung der Pest in der Antike und in der Romania, p. 44-55; M. Gale, Myth and Poetry in Lucretius, p. 112-114 et 225-228; K. Stoddard, "Thucydides, Lucretius and the end of de De rerum natura». Plus spécifiquement sur Thucydide: J. Grimm, Die literarische Darstellung der Pest in der Antike und in der Romania, p. 31-44; D. L. Page, "Thucydides' Description of the Great Plague at Athens", p. 97-118; A. Gervais, "A propos de la "Peste" d'Athènes: Thucydide et la littérature de l'épidémie», p. 409-411.

27. P. Demont, "La Peste: un inédit d'Albert Camus, lecteur de Thucydide», p. 139140 ; W. Schmitz, "Diagnosen der "Pest” in Athen (430-426 v. Chr.)».

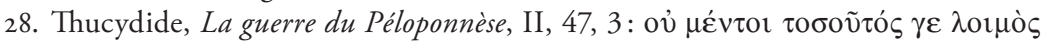

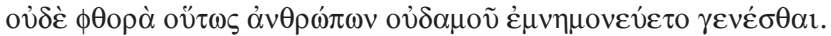

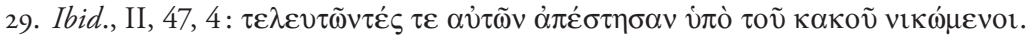




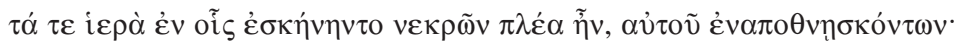

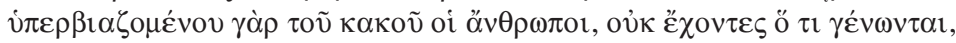

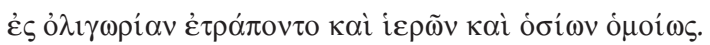

Les lieux sacrés où l'on campait étaient pleins de cadavres, car on mourait sur place: devant le déchaînement du mal, les hommes, ne sachant que devenir, cessèrent de rien respecter, soit de divin, soit d'humain ${ }^{30}$.

Ou encore:

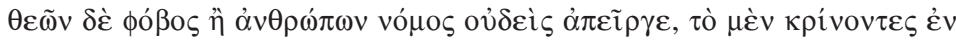

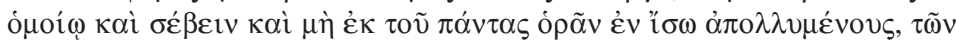

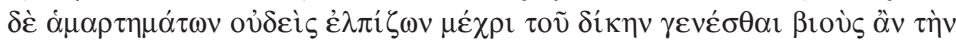

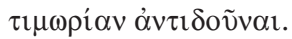

Crainte des dieux ou loi des hommes, rien ne les arrêtait: d'une part, on jugeait égal de se montrer pieux ou non, puisque l'on voyait tout le monde périr semblablement, et, en cas d'actes criminels, personne ne s'attendait à vivre assez pour que le jugement eût lieu et qu'on eût à subir sa peine ${ }^{31}$.

Thucydide dépeint alors les gens ensevelissant les cadavres à la sauvette ou plaçant les corps des leurs sur les bûchers des autres. Son récit procède du même souci scientifique qui préside à la composition de toute son œuvre; il est fait avec l'objectivité qui le caractérise, et cela en dépit du fait qu'il avait lui-même été atteint de la maladie ${ }^{32}$. Reconnaissant son ignorance quant à l'origine et aux causes de l'épidémie, l'historien veut s'attacher à en dépeindre les symptômes, pour pouvoir «si jamais elle se reproduisait, profiter d'un savoir préalable et n'être pas devant l'inconnu" ${ }^{33}$. Nulle part, il n'évoque la possibilité que le fléau soit la conséquence d'un châtiment divin. Il rompt avec la représentation religieuse des épidémies, traditionnellement interprétées comme résultant du nonrespect des rites de sépulture. Chez lui, c'est l'inverse qui se produit.

3o. Ibid., II, 52, 3 .

3I. Ibid., II, 53, 4.

32. A. Gervais ("A propos de la "Peste” d'Athènes: Thucydide et la littérature de l'épidémie», p. 397) souligne chez Thucydide «la sobriété du propos et une objectivité qui se donne parfois un air d'insensibilité».

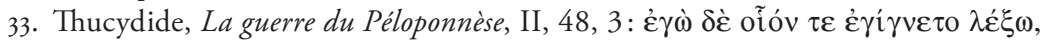

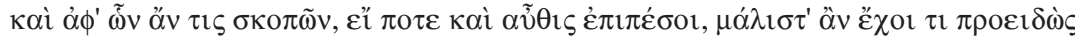

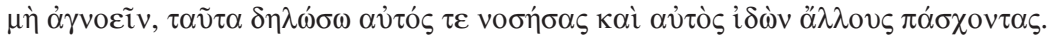


Pour citer Paul Demont, «la peste cause le crime, au lieu d'en être la conséquence ${ }^{34}$.

On voit bien les éléments qui ont pu inciter Lucrèce à s'inspirer de ce texte pour peindre son propre tableau de la peste. Le récit de Thucydide présentait une situation exceptionnelle et, pour reprendre une formule d'Alice Gervais, "transcend[ait] son actualité du moment, pour revêtir une valeur universelle» ${ }^{35}$. L'épidémie désagrégeait les normes de la collectivité; la peur de la mort, la peur de l'inconnu, l'incapacité d'expliquer les causes de la maladie, la perversion des rites religieux, l'absence des dieux, tout cela offrait au poète matière à illustrer les principes de la philosophie épicurienne. De plus, l'événement s'était produit à Athènes, ville où, selon Lucrèce, Epicure naîtrait un siècle plus tard - un siècle trop tard. Sur certains points, le poète latin supplée au récit de Thucydide en offrant l'explication physique des causes de l'épidémie que l'historien ne pouvait donner. Mais en même temps, Lucrèce «déshistoricise» la peste d'Athènes: il n'est plus question de la guerre et l'adverbe quondam (autrefois) remplace les indications chronologiques précises de l'historien. S'il emprunte au récit historique plusieurs traits saisissants, il les transpose dans le langage de la poésie: l'impuissance des médecins sobrement décrite par Thucydide prend ainsi une ampleur emphatique à travers la personnification métonymique de la science médicale:

Nec requies erat ulla mali : defessa iacebant corpora. Mussabat tacito medicina timore, quippe patentia cum totiens ardentia morbis lumina uersarent oculorum expertia somno.

Nul répit dans le mal; épuisés, les corps gisaient immobiles. Saisie d'une crainte muette, la médecine ne savait que balbutier, tandis que le malade tournait sans cesse vers elle ses yeux grands ouverts, enflammés par la fièvre, privés de tout sommeil ${ }^{36}$.

34. P. Demont, «La Peste: un inédit d'Albert Camus, lecteur de Thucydide», p. 143. 35. A. Gervais, "A propos de la "Peste" d'Athènes: Thucydide et la littérature de l'épidémie», p. 395.

36. Lucrèce, De la Nature, VI, 1178-1181. 
De même, la description des corps accumulés dans les temples, celle des cadavres jetés sur les bûchers des autres, sont amplifiées pour faire de la "peste» d'Athènes, comme on l'a dit, un mythe philosophique ${ }^{37}$.

Mon cheminement aurait pu se poursuivre, remonter jusqu'à Homère, s'arrêter chez le poète Ovide, chez Sénèque, chez les historiens Tite Live, Ammien Marcelin ou Procope. J'aurais pu évoquer le Decameron de Boccace, qui s'ouvre sur la description de l'épidémie de peste ayant ravagé Florence en 1348. Un débat s'est engagé entre les savants sur la question de la source de Boccace: Thucydide ou Lucrèce? Ou Thucydide à travers Lucrèce? Ou Lucrèce à travers Virgile, Ovide, Sénèque ou Macrobe? ${ }^{38}$ Une tradition s'était constituée autour du thème de la peste et certains motifs récurrents s'étaient transmis à travers le temps. J'aurais pu parler aussi du rôle de l'école dans cette transmission. A travers les différentes étapes de leur formation, les élèves étaient entraînés à lire les auteurs classiques, à les apprendre par cœur, à les commenter et à les paraphraser pour s'imprégner de leur style et de leur génie. Ils devaient aussi se plier à une série d'exercices, comme la description. Or parmi les sujets de descriptions mentionnés dans les ouvrages rhétoriques, on trouve l'épidémie ${ }^{39}$. Dans les déclamations, qui sont des discours judiciaires fictifs formant l'élève à plaider le pour et le contre d'un cas donné, on rencontre le thème de l'épidémie. Son traitement donne lieu à des développements pathétiques censés éveiller la pitié de l'auditoire. Ici, il n'est pas question de considérations éthiques, philosophiques ou religieuses; ce sont des exercices de style sur des motifs topiques, susceptibles d'être réutilisés à d'autres occasions et dans d'autres compositions ${ }^{40}$.

Pour conclure mon parcours et revenir aux temps modernes, j'aimerais évoquer brièvement la récriture qu'offre de ce thème Albert Camus, un auteur mentionné (entre autres) par Alice Gervais célébrant avec emphase «l'inspiration de l'Occident [qui] ne cesse pas de s'abreuver aux

37. M. Gale, Myth and Poetry in Lucretius, p. 225.

38. G. Getto, «La peste del "Decameron" e il problema della fonte lucreziana».

39. Cf. les prescriptions du rhéteur Théon dans l'édition des Rhetores Graeci, II, de L. Spengel (Teubner), p. 118.

40. Voir ce qu'en dit Encolpe au début du Satiricon de Pétrone (ch. 1); Quintilien, Institution oratoire, II, 10, 4-5; Ps. Quintilien, Déclamations mineures, 326 et 384; Calpurnius Flaccus, Déclamations, 19, 43. Sur les déclamations, les thèmes traités et leurs descriptions volontiers horrifiques, je me permets de renvoyer à D. van MalMaeder, La fiction des déclamations. 
sources de l'ancienne Hellade ${ }^{41}$. La Peste de Camus relate des événements qui se sont produits en $194^{*}$, à Oran, ville frappée par une épidémie de peste bubonique. Le roman fut publié en 1947, au lendemain de la seconde guerre mondiale. Dans un lettre à Roland Barthes, datant du 11 janvier 1955, Camus écrit:

La peste, dont j'ai voulu qu'elle se lise sur plusieurs portées, a cependant comme contenu évident la lutte de la résistance européenne contre le nazisme ${ }^{42}$.

L'un des personnages principaux de ce roman est le père Paneloux, un jésuite, qui attribue au fléau une origine divine, se faisant la voix de la tradition antique. Dans son premier sermon, il s'exclame ainsi: «Mes frères, vous êtes dans le malheur, mes frères, vous l'avez mérité. » Confronté au spectacle de l'agonie d'un enfant, il se montrera plus humble, convenant que «cela est révoltant parce que cela passe notre mesure. Mais peut-être devons-nous aimer ce que nous ne pouvons pas comprendre." Face à lui, le docteur Rieux, qui se fait "une autre idée de l'amour" et se refuse «jusqu'à la mort d'aimer cette création où des enfants sont torturés " 43 . L'inexistence de Dieu, telle est la conclusion à laquelle il aboutit, radicalisant ainsi le discours sur les dieux de Lucrèce. De fait, comme Paul Demont l'a montré, Camus s'inspira tant de Lucrèce que de Thucydide et puisa chez eux certains motifs et idées pour leur donner un sens nouveau en accord avec sa propre vision de l'homme et du monde ${ }^{44}$. Si le texte définitif de La Peste n'offre que peu de points de comparaison avec ces deux auteurs, Camus avait imaginé, dans une première version, faire tenir un journal de la peste à un "professeur de latin-grec», Philippe Stephan, témoin de première main puisque, comme Thucydide, il avait lui-même été atteint de la maladie et en avait réchappé. Dans son article, Paul Demont cite quatre feuilles inédites figurant dans le premier état de la première version de La Peste, déposé à la Bibliothèque nationale

4I. A. Gervais, "A propos de la "Peste” d'Athènes: Thucydide et la littérature de l'épidémie», p. 429.

42. Lettre ouverte à Roland Barthes parue en février 1955 dans Club, Bulletin du Club du meilleur livre; cette lettre se lit dans les Euvres complètes d'Albert Camus, p. 285-287.

43. Ces citations se trouvent aux pages 98 et 184 de l'édition de La Pléiade.

44. P. Demont, "La Peste: un inédit d'Albert Camus, lecteur de Thucydide", p. 144-154. 
de France, qui contiennent un commentaire de Stephan sur Thucydide et Lucrèce ${ }^{45}$. A propos du texte de ce dernier (dont il abrège le nom en Luc), le "professeur de latin-grec" note qu'il "n'est jamais qu'un commentaire de celui de Thucydide», avant d'ajouter:

Luc commente et il n'est pas mauvais que nous commentions à notre tour. Ce n'est pas trop de deux commentaires sur un texte aussi important. Les chrétiens depuis des siècles vivent au milieu d'une cascade de commentaires de commentaires. Pourquoi pas les pestiférés. C'est une religion qui en vaut bien une autre.

Si le personnage de Stephan est resté à l'état d'esquisse, cédant la place dans la version définitive au docteur Rieux (qui incarne en quelque sorte l'impuissance de la médecine dont parlaient Thucydide et Lucrèce), sa profession de foi en forme de boutade n'en illustre pas moins de façon idéale le principe de pérennité de la culture antique que j’ai tenté de mettre en évidence ici.

Ce commentaire m'amène à ma conclusion, que je voudrais sans emphase, et qui renverra à l'épigraphe: aussi longtemps qu'il y aura des gens pour lire ces textes qui nous ont été transmis à travers les millénaires, aussi longtemps que ces textes inspireront de nouvelles créations et qu'ils nous amèneront à réfléchir, indépendamment de toute considération matérielle, à la condition humaine, aux rapports avec Dieu ou la nature, ces textes demeureront des événements.

Danielle van MaL-Maeder

Université de Lausanne

45. Le commentaire de Stephan sur Thucydide et Lucrèce se lit désormais dans les Euvres complètes d'Albert Camus, p. 258-266. 


\section{BIBLIOGRAPHIE}

\section{Textes}

Endelechius, De la mort des boufs, in Antologia latina I, 2, ed. Franciscus Buecheler et Alexander Riese, Leipzig, Teubner, 1906, p. 334-339.

LuCrèce, De la nature, texte établi et traduit par Alfred Ernout, 2 vols, Paris, Les Belles Lettres, 1985-2002.

Thucydide, La guerre du Péloponnèse, texte établi et traduit par Jacqueline de Romilly, Paris, Les Belles Lettres, 1953.

Virgile, Bucoliques, texte établi et traduit par Eugène de Saint-Denis, Paris, Les Belles Lettres, 1978.

—, Géorgiques, texte établi et traduit par Eugène de Saint-Denis, Paris, Les Belles Lettres, 1995.

\section{Etudes}

Barton, Monika, Spätantike Bukolik zwischen paganer Tradition und christlicher Verkündigung. Das Carmen De mortibus boum des Endelechius, Trier, Wissenschaftlicher Verlag Trier, 2000.

BüChner, Karl, «Die Pest. Ihre Darstellung bei Thukydides, Lukrez, Montaigne, Camus", in Humanitas Romana. Studien über Werke und Wesen der Römer, hrsg. von Karl Büchner, Heidelberg, Carl Winter Universitätsverlag, 1957, p. 64-79.

Camus, Albert, Euvres complètes, Paris, La Pléiade, 2006.

Citroni, Mario, "Percezioni di classicità nella letteratura latina», in Che cos'è il classicismo?, a cura di Roberto Cardini e Mariangela Regoliosi, Roma, Bulzoni editore, 1998, p. 1-34.

Clausen, Wendell, A Commentary on Virgil Eclogues, Oxford/New York, Clarendon Press/Oxford University Press, 1994. 
Cox, Fiona, "Envoi: the death of Virgil», in The Cambridge Companion to Virgil, ed. Charles Martindale, Cambridge, Cambridge University Press, 1997, p. 327-336.

Curtius, Ernst Robert, La littérature européenne (traduit de l'allemand par Jean Bréjoux), Paris, Presses universitaires de France, 1956.

Demont, Paul, "La Peste: un inédit d'Albert Camus, lecteur de Thucydide", Antike \& Abendland, 42 (1996), p. 137-154.

Effe, Bernd et Binder, Gerhard, Antike Hirtendichtung. Eine Einführung, Düsseldorf/Zürich, Artemis, 2001.

Freudenburg, Kirk, "Lucretius, Vergil, and the Causa Morbi», Vergilius, 33 (1987), p. 59-74.

Gale, Monica, "Man and Beast in Lucretius and the Georgics", The Classical Quarterly, 41 (1991), p. 414-426.

-, Myth and Poetry in Lucretius, Cambridge/New York, Cambridge University Press, 1994.

-, Virgil on the Nature of Things: the Georgics, Lucretius and the Didactic Tradition, Cambridge/New York/Melbourne/Madrid, Cambridge University Press, 2000.

Gervais, Alice, "A propos de la "Peste" d'Athènes: Thucydide et la littérature de l'épidémie", Bulletin de l'Association Guillaume Budé, 1972, p. 395-429.

Getto, Giovanni, «La peste del "Decameron" e il problema della fonte lucreziana", Giornale storico della letteratura italiana, 135 (1958), p. 507-523.

Green, Roger, "Refinement and Reappraisal in Vergilian Pastoral», in Romane memento. Vergil in the Fourth Century, ed. Roger Rees, London, Duckworth, 2004, p. 17-32.

Grimm, Jürgen, Die literarische Darstellung der Pest in der Antike und in der Romania, München, Wilhelm Fink, 1965.

Hardie, Philip, "Lucretius and later Latin literature in antiquity», in The Cambridge Companion to Lucretius, eds Stuart Gillespie and Philip Hardie, Cambridge, Cambridge University Press, 2007, p. 111-127.

Holzberg, Niklas, Vergil. Der Dichter und sein Werk, München, C. H. Beck, 2006, p. 14-18.

Mal-Maeder, Danielle van, La fiction des déclamations, Leiden/Boston, Brill, 2007. 
Martindale, Charles, Redeeming the text. Latin poetry and the hermeneutics of reception, Cambridge/New York, Cambridge University Press, 1993.

—, "Introduction: "The classic of all Europe" ", in The Cambridge Companion to Virgil, ed. Charles Martindale, Cambridge, Cambridge University Press, 1997, p. 1-18.

-, "Green politics: the Eclogues", in The Cambridge Companion to Virgil, ed. Charles Martindale, Cambridge, Cambridge University Press, 1997, p. 107-124.

Mayer, Roland, "Latin Pastoral after Vergil», in Brill's Companion to Greek and Latin Pastoral, eds Marco Fantuzzi and Theodore Papanghelis, Leiden/Boston, Brill, 2006, p. 451-466.

Mynors, Roger Aubrey Baskerville, Virgil. Georgics, Oxford, Clarendon Press, 1990.

Page, Denys Lionel, "Thucydides' Description of the Great Plague at Athens", Classical Quarterly, 3 (1953), p. 97-119.

Perutelli, Alessandro, "Bucolics", in A Companion to the study of Vergil, ed. Nicholas Horsfall, Leiden/New York/Köln, Brill, 1995, p. 27-62.

Powell, Anton, Virgil the Partisan. A study in the re-integration of Classics, Swansea, The Classical Press of Wales, 2008.

Reverdin, Olivier et Grange, Bernard (éds), Le clacissisme à Rome aux $I^{\text {ers }}$ siècles avant et après J.-C., Entretiens sur l'Antiquité classique tome XXV, Fondation Hardt, Vandoures-Genève, 1978.

Schmid, Wolfgang, "Tityrus Christianus. Probleme religiöser Hirtendichtung an der Wende vom vierten zum fünften Jahrhundert", Rheinisches Museum, 96 (1953), p. 101-165.

Schmitz, Winfried, "Diagnosen der "Pest" in Athen (430-426 v. Chr.)", in Pest. Die Geschichte eines Menschheitstraumas, hrsg. von Mischa Meier, Stuttgart, Klett-Cotta, 2005, p. 44-65.

Stoddard, Kate, "Thucydides, Lucretius and the end of the De rerum natura", Maia, 48 (1996), p. 107-128.

TARrant, Richard John, "Aspects of Vergil's reception in antiquity", in The Cambridge Companion to Virgil, ed. Charles Martindale, Cambridge, Cambridge University Press, 1997, p. 56-72.

—, "Poetry and power: Virgil's poetry in contemporary context", in The Cambridge Companion to Virgil, ed. Charles Martindale, Cambridge, Cambridge University Press, 1997, p. 169-187. 
West, David A., "Two Plagues: Virgil, Georgics 3, 478-566 and Lucretius 6, 1090-1286", in Creative Imitation and Latin Literature, eds David West and Tony Woodman, Cambridge/ London/New York/Melbourne, Cambridge University Press, 1979, p. 71-88. 
